\title{
Local and Systemic Recurrence is the Achilles Heel of Cancer Surgery
}

\author{
Louis A. Aliperti ${ }^{1}$, Jarrod D. Predina ${ }^{1}$, Anil Vachani ${ }^{2}$, and Sunil Singhal ${ }^{1}$ \\ ${ }^{1}$ Division of Thoracic Surgery, Department of Surgery, University of Pennsylvania School of Medicine, Philadelphia, PA; \\ ${ }^{2}$ Division of Pulmonary, Allergy and Critical Care, Department of Medicine, University of Pennsylvania School of \\ Medicine, Philadelphia, PA
}

This year approximately 569,490 Americans, more than 1,500 people per day, are expected to die of cancer. Cancer has supplanted heart disease as the leading cause of death in the United States in men and women younger than age 85 years (American Cancer Society, Facts and Figures 2010). Cancer also has a major economic impact on the U.S. economy, with 2010 estimates of cancer associated costs of $\$ 263.8$ billion (American Cancer Society, Facts and Figures 2010). The four most common cancers (lung, colorectal, prostate, and breast cancer) account for more than half of all cancer incidences in the United States. Surgery, chemotherapy, and radiotherapy are the most established therapies for patients with these malignancies (American Cancer Society, Facts and Figures 2010). Surgery continues to be the most effective therapy for cancer in the United States. It is several-fold more effective than chemotherapy and radiation in curing patients with cancer. Unfortunately, many people are not surgical candidates due to advanced stage disease or comorbid conditions.

During the past two decades, both mortality and observed cancer survival statistics have improved dramatically both in patients who do and do not undergo surgery. This likely reflects an improvement in cancer care in the United States. Since 1973, the Survival Epidemiology and End Results (SEER) Program sponsored by the National

Louis A. Aliperti and Jarrod D. Predina contributed equally to this work.

(C) Society of Surgical Oncology 2010

Published Online: 16 December 2010

S. Singhal

e-mail: sunil.singhal@uphs.upenn.edu
Cancer Institute has kept a publicly accessible database that has catalogued the outcome of cancer patients (Surveillance, Epidemiology, and End Results Program. Bethesda, SEER Stat Database). These data include information regarding incidence and mortality, as well as the initial surgical and adjuvant therapy provided for the treatment of cancer. This improvement in survival and mortality in this database during the past 20 years is likely due to better screening and detection, biologically targeted systemic therapies, improved surveillance, improved surgical methods, improved patient selection, and more effective adjuvant therapies. Cancer surveillance also has improved dramatically with the improvement in preoperative imaging and minimally invasive and noninvasive staging techniques, such as mammography, colonoscopy, endobronchial ultrasound, and prostate-specific antigen testing.

The goal of this short discussion is to highlight some of the trends that have been observed in cancer and surgery during the past 20 years and the role that surgery continues to play in the oncologic community. We found that surgery remains an effective therapy for solid tumors in the United States and dramatically improves survival rates for patients with solid tumors. The proportion of cancer patients who undergo surgery has declined during the past 20 years for all major cancer types. Additionally, we found that local and systemic recurrences continue to be the Achilles heel of Surgical Oncology; although surgery does improve survival, almost one-third of surgical patients will ultimately recur locally and/or systemically. This is an important reminder to surgeons that we must work diligently to improve cancer care for our patients preoperatively, intraoperatively, and postoperatively to prevent these recurrences. Research and clinical trials should be focused on this major objective in the coming decades. 


\section{CANCER SURVIVAL HAS IMPROVED DURING THE PAST 20 YEARS}

In the United States, surgical resection currently offers the best opportunity to cure solid tumor malignancies. On reviewing the SEER database from 2000-2004, we found a total of 777,136 of 1,190,322 registered patients with solid organ cancers who underwent surgery. When examining postoperative 5-year survivals for the four most commonly diagnosed cancers, Kaplan-Meier analysis demonstrates significant survival benefits for patients qualifying for surgery resection (Fig. 1a). The most dramatic increase in observed survival was seen in lung cancer, with an increase in survival for surgical versus nonsurgical cohorts of 11fold at 5 years (Fig. 1b). Increases of 3.7- and 2.5-fold were seen in modern colorectal and breast cancer cohorts, respectively, whereas only a 1.2 -fold increase was noted in contemporary prostate cancer cohorts (Fig. 1b). A weighted average of patients who underwent surgery for these malignancies yielded a 5-year survival of 74.8 versus
$42.1 \%$ in nonsurgical patients. It is important to interpret these survival benefits with the understanding that various factors, including histology, stage, and comorbidities, influence surgical candidacy, and likely confound longterm outcomes.

Clearly, the importance of surgery's role in the oncology community is well established; however, the proportion of patients with cancer undergoing surgery for lung, breast, colorectal, and prostate cancers has decreased during the last 20 years. From 1983-1987, 71.6\% of patients diagnosed with these tumors were treated surgically versus $65.3 \%$ of those patients diagnosed from 2000-2004. The greatest decrease in proportion was $17 \%$ decrease observed in lung cancer, whereas breast cancer only slightly decreased by $1.1 \%$. Prostate and colorectal cancers had an overall decrease in the use of surgical intervention of 11.4 and 3\%, respectively. These data almost certainly suggest improvements in nonsurgical therapies (such as targeted biological therapies, chemotherapy, and radiation therapy), along with an improvement in diagnostic imaging and
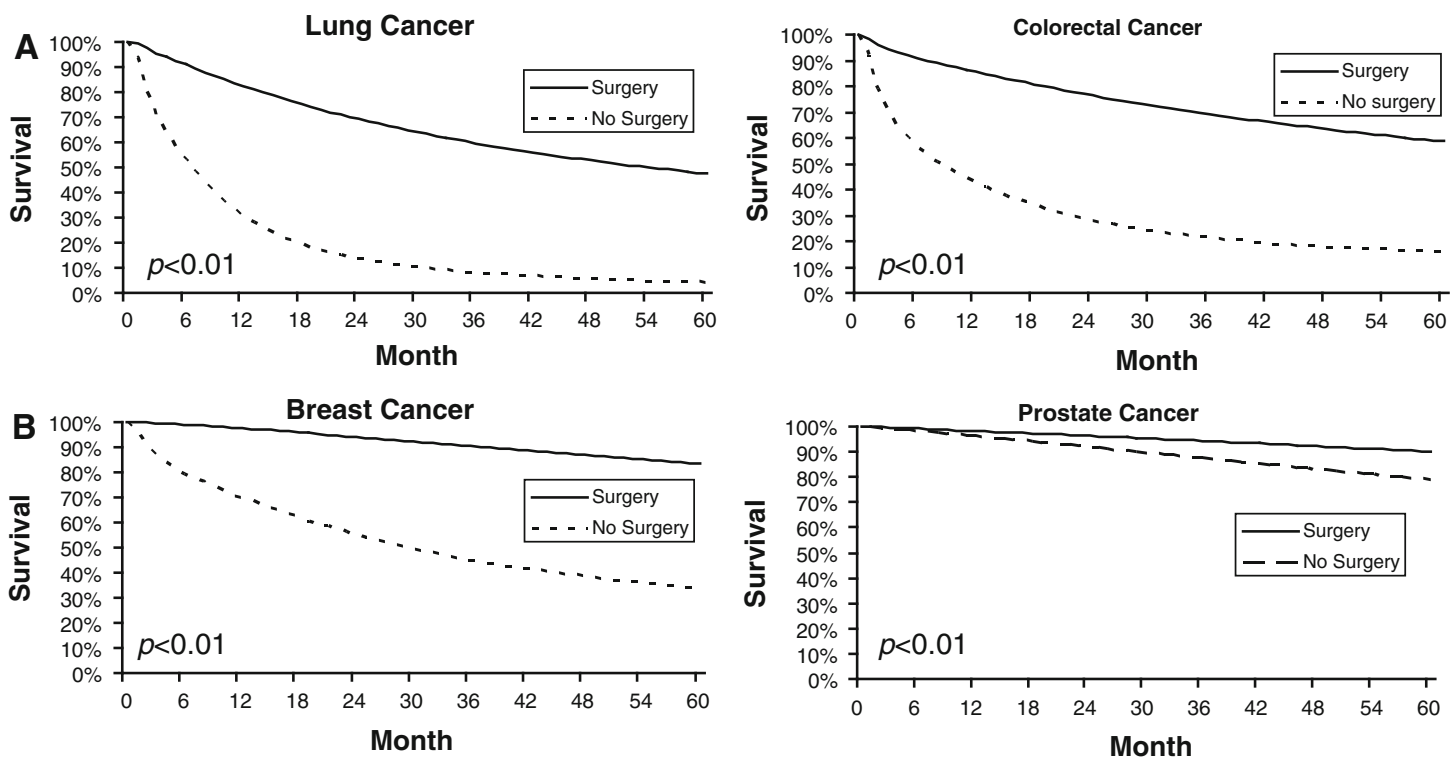

\begin{tabular}{|c|c|c|c|c|}
\hline & $\begin{array}{c}\text { Observed 5 Year } \\
\text { Survival With } \\
\text { Surgery (\%) }\end{array}$ & $\begin{array}{c}\text { Observed 5 Year } \\
\text { Survival Without } \\
\text { Surgery (\%) }\end{array}$ & $\begin{array}{c}\text { Fold Increase } \\
\text { in Survival } \\
\text { With Surgery* }\end{array}$ & $\begin{array}{c}\text { Observed 5 Year } \\
\text { Survival for All } \\
\text { Diagnosed (\%) }\end{array}$ \\
\hline Lung & 47.1 & 4.3 & 11 & 13.9 \\
\hline Colorectal & 58.4 & 16.0 & 3.7 & 53.5 \\
\hline Breast & 83.4 & 33.2 & 2.5 & 80.5 \\
\hline Prostate & 90.0 & 78.2 & 1.2 & 82.6 \\
\hline
\end{tabular}

FIG. 1 Representation of 5-year survival among patients diagnosed with lung, colorectal, breast, and prostate cancers. Data were obtained from SEER database using SEER*Stat software (a) Kaplan-Meier analysis demonstrating survival benefits observed in patients undergoing surgery versus no surgery. (b) Tabulation of survival statistics for those diagnosed with lung, colorectal, breast, and prostate cancers. *Values were calculated using the following equation: (observed 5-year survival with surgery)/(observed 5-year survival without surgery) 
testing that may reveal metastasis preoperatively thereby eliminating previously acceptable surgical candidates. Finally, changes in referral patterns and more aggressive medical and radiation oncology communities have had subtle effects in this finding.

Interestingly, on querying the SEER database using SEER*Stat (a statistical program that allows for specific searching of the SEER database), cancer survival of those qualifying for surgery was noted to have improved during the same time period. The most noteworthy improvements were in prostate cancer, which had an observed 5-year survival of $59.7 \%$ for surgical patients during the 1983-1987 interval, which improved to almost 90\% during the 2000-2004 interval. Lung cancer patients who undergo surgery also improved their observed survival from 36.6 to $47.1 \%$ - a $28.7 \%$ increase. Breast and colorectal cancer had improvements of 12.9 and $15 \%$, respectively. These findings further support the essential role that surgery continues to play in the oncology field. Additionally, these findings give credence to the notion that tremendous strides have been made in screening/surveillance protocols, identification of optimal surgical candidates, and development of more potent systemic therapies, which can be effectively utilized as surgical adjuvants.

\section{CANCER RECURRENCE REMAINS THE BIGGEST CHALLENGE IN PATIENTS UNDERGOING SURGERY}

The SEER database does not track information regarding cancer recurrences after curative surgery. We, thus, performed a focused literature review of published recurrence data from large reports. In searching for literature, the keywords "recurrence AND postoperative" were used. Only papers published during the past 7 years were included in our search. We ultimately selected the largest series for each cancer. Although this may not be truly representative, it provides a framework for discussion. Our review found a common trend among these four cancers: patients diagnosed with postoperative recurrence, regardless of being local or systemic, experience poorer 5-year survival compared with surgical candidates who do not recur. This is likely a result of these patients already having received adjuvant chemotherapy and/or radiation, or requiring second-line or salvage therapy. In addition, these patients performance status often decline after surgery and adjuvant therapy, making it more likely to have a complication from second-line or salvage therapy.

When examining long-term outcome after surgery for lung cancer, we consulted two recent, large-scaled studies that were conducted at major U.S. academic centers. ${ }^{1,2}$ The largest report by Sugimura et al., a 4-year study following
1,073 patients treated surgically for non-small-cell lung cancer at the Mayo Clinic in Rochester, MN, documented that $41.5 \%$ of patients recurred postoperatively (Fig. 2a). Of these, $43.8 \%$ recurred locally only and $56.2 \%$ recurred distantly (Fig. 2b). ${ }^{1}$ The other study, which was completed by Kelsey and colleagues at Duke University, focused on early-stage NSCLC and yielded similar recurrence rates of $36 \%{ }^{2}$ The pattern of recurrence, however, was slightly different with $25 \%$ of recurrences occurring locally only and $75 \%$ occurring distantly. ${ }^{2}$

For these patients with postoperative lung cancer recurrences, the survival was dismal. According to $\mathrm{Su}-$ gimura's report, the overall 2- and 5-year survivals for this recurrent cohort were found to be 17 and $12 \%$, respectively (Table 1). ${ }^{1}$ This indicates that postoperative recurrences in lung cancer frequently occur early, leading to poor outcome. Of note, the author commented that additional treatment was not an option in approximately a third of patients due to rapid disease progression. ${ }^{1}$

To analyze postoperative recurrences in colorectal cancer, we first reviewed a recent study completed by Tsikitis and colleagues, which reported on 791 patients involved in the National Cancer Institute's Clinical Outcomes of
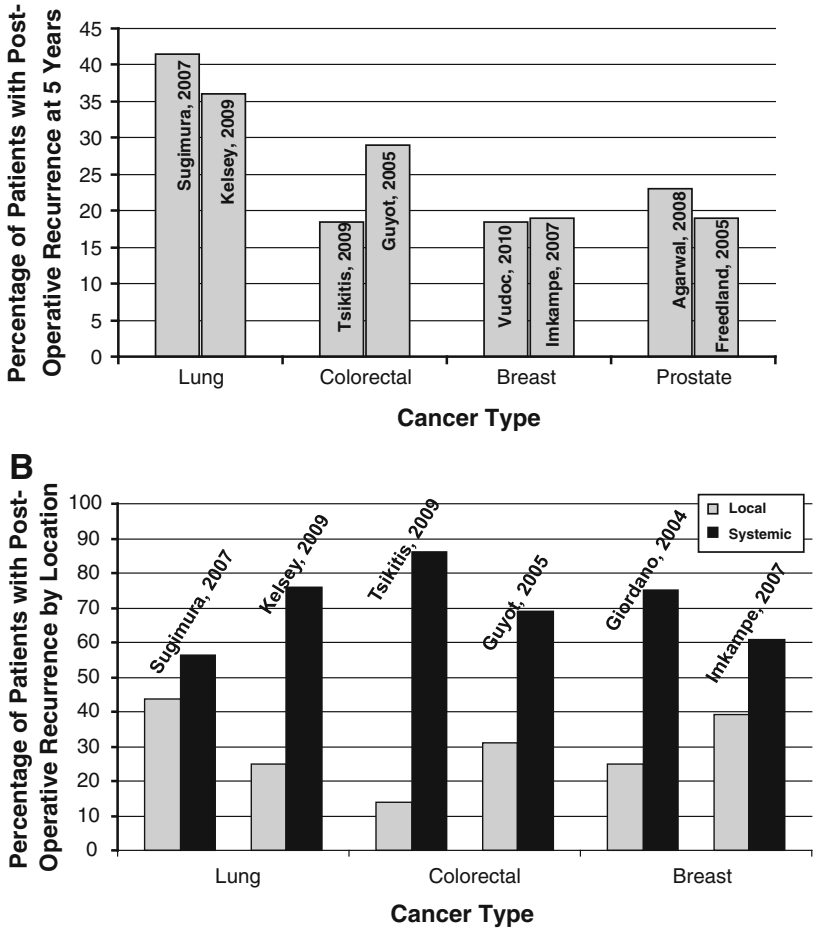

FIG. 2 Postoperative recurrence data for lung, colorectal, breast and prostate cancers. Data were obtained from focused literature review. a Percentage of patients undergoing surgery who are subsequently diagnosed with cancer recurrence within 5 years of initial surgical resection. b Postoperative recurrences by location: local versus systemic 
TABLE 1 Five-year postoperative survival after surgery

\begin{tabular}{lll}
\hline & $\begin{array}{l}\text { Five-year survival } \\
\text { following surgery } \\
(\%)^{\text {a }}\end{array}$ & $\begin{array}{l}\text { Five-year survival } \\
\text { following surgery } \\
\text { with recurrence }(\%)^{\mathrm{b}}\end{array}$ \\
\hline Lung & 47 & 12 ; Ref. ${ }^{1}$ \\
Colorectal & 58 & 26 Ref. $^{4}$ \\
Breast & 83 & 26 Ref. $^{7}$ \\
Prostate & 90 & 93 Ref. $^{9}$
\end{tabular}

${ }^{a}$ Includes both survivorship of those with and without recurrences. Data obtained from SEER database

b Includes only survival data of those with postoperative cancer recurrences. Estimations based on literature review

Surgical Therapy Trial. ${ }^{3}$ The overall recurrence rate was found to be $18.5 \%$ (Fig. 2a). ${ }^{3}$ From the data presented in the Tsikitis paper, we estimate that approximately $14 \%$ of these recurrences occurred locoregionally (at the site of resection or at a local lymph node), whereas approximately $86 \%$ of recurrences occurred distantly (Fig. 2b). ${ }^{3}$ A report by Guyot and colleagues, which describes the follow-up of more than 3,600 postoperative colorectal patients, supports the Tsikitis report: the overall recurrence rate was approximately $29 \%$, with $31 \%$ occurring locally with $69 \%$ occurring distantly (Fig. 2a, b). ${ }^{4}$ According Guyot's report, recurrences for colorectal cancer also is associated with decreases in postoperative survival. ${ }^{4}$ The overall 5-year survival rate was $21.6 \%$ for those presenting systemic recurrences and $31.6 \%$ for those with local recurrences. ${ }^{4}$ After appropriate weighting, the 5-year survival after recurrence for colorectal cancer was estimated to be $26.9 \%$ (Table 1).

Literature review analyzing recurrences and long-term outcomes after breast cancer surgery revealed a similar trend. First, a Canadian study conducted by Voduc et al. describes a cohort of 4,033 women who received breastconserving surgery (BCS) or total mastectomy between 1986 and $1992 .{ }^{5}$ The combined recurrence rate was $18.5 \%$ (Fig. 2a). ${ }^{5}$ A second study conducted by Giordano and colleagues at M.D. Anderson Cancer Center describes 834 women who developed breast cancer recurrences between 1974 and 2000. ${ }^{6}$ According to Giordano's work, the dominant site of recurrence was local in $24.8 \%$ of patients, whereas almost $75 \%$ of patients had a dominant site that was systemic (Fig. 2b). ${ }^{6}$ Finally, a study following 1,558 patients in England between 1989 and 2003 revealed a recurrence rate of $18.9 \%$, supporting Voduc's study (Fig. 2a). ${ }^{7}$ This study, completed by Imkampe and colleagues, breaks the site of recurrence into four groups: local, lymph node, bone, and visceral. For the purposes of discussion and comparison, we consider local and nodal recurrence to be local and bone and visceral recurrences to be distant. ${ }^{7}$ Using this methodology, $39 \%$ of recurrences occurred locally versus $61 \%$ distantly (Fig. 2 b). ${ }^{7}$
As in colon cancer, survival postrecurrence in breast cancer is highly dependent on the location of recurrence. The 3-year survival in recurrent breast cancer was shown to be $83 \%$ for patients who recurred locally. ${ }^{7}$ Regional and systemic recurrence dramatically decrease 3 -year survivals to $33 \%$ for patients with nodal recurrence, $23 \%$ for patients with bone recurrence, and $13 \%$ for visceral recurrences, including lung, liver, peritoneally, or the nervous system. ${ }^{7}$ Combined, the estimated 3-year survival and 5-year survivals were estimated to be 37 and $26 \%$, respectively (Table 1$)^{7}$

The primary means of surveillance in prostate cancer, both prediagnosis and posttherapy, has become the Prostate-Specific Antigen (PSA) test. Clinicians frequently refer to biochemical recurrence in prostate cancer patients, and much of the literature focuses on biochemical recurrence. In a recent long-term study published by Agarwal et al. of Case Western Reserve, of 4,342 men who underwent radical prostatectomy, 1,003 or $23.1 \%$ recurred biochemically (Fig. 2a). ${ }^{8}$ In a second study by Freedland and colleagues at Johns Hopkins, almost 19\% of those undergoing radical prostatectomy had evidence of biochemical recurrence (Fig. 2b). ${ }^{9}$

In conclusion, although all surgeons hope to cure all of their patients, local and systemic recurrences remain the main failure after surgery. Based on our literature search, as many as 20 to $40 \%$ of patients will recur after surgery. Overall, approximately 621,709 patients in the United States undergo surgery annually, and based on our study of the four most common cancers, we extrapolate that somewhere between 120,000 and 250,000 patients will suffer from a postoperative recurrence of their disease.

\section{CONCLUSIONS}

Currently, in the United States, cancer patients are treated with surgery, chemotherapy, or radiation, or a combination of these approaches. Surgery is the single most effective therapy for these cancers. For most cancers, survival for patients who undergo surgery increases from two- to tenfold compared with the same patients who are not surgical candidates. While this may reflect the inherent benefits of cytoreduction, we must remember that surgical candidates are often diagnosed at an early stage and have more favorable prognoses than nonsurgical patients (a lead time bias).

During the past 20 years, the number of patients who qualify for surgery has decreased. This is a result of improved preoperative metastatic workup, alternative treatment strategies, and referral patterns in the United States. Despite this decrease in patients who qualify for surgery, survival after surgery has improved dramatically 
for a variety of reasons. One explanation is due to improved preoperative staging. Advances in this area account for those patients who were previously thought to have early-stage disease but are discovered to have advanced stage disease, ultimately do not undergo surgery. Secondly, improved screening regimens (colonoscopy, mammography, digital rectal exam, etc.) are better equipped to detect malignancies at early stages, which sets the stage for better postoperative outcomes. Finally, adjuvant therapies have improved significantly in all cancer types, most notably lung cancer and breast cancer.

The most important challenge to this surgical community is preventing recurrences. Recurrences tend to be the most common cause of failure in our community. Different cancers tend to favor local versus systemic recurrence. This is largely related to disease biology and less likely to intraoperative issues.

Even in those patients who develop recurrences after surgery, 5-year survival after recurrence is still higher than for those patients who did not initially qualify for surgery. Again, this may reflect the stage or histology of the disease at the initial presentation. This data suggest that oncology surgeons have a clear opportunity for significant improvement in our field, specifically in preventing recurrences.

Surgeons have the opportunity to influence care in three areas for their patients: preoperatively, intraoperatively, and postoperatively. Research and support of surgical investigations must focus on all aspects of patient care. Better staging and assessment preoperatively combined with neoadjuvant therapies for cancer subsets must continue to be evaluated in rigorous clinical trials. Intraoperative techniques to examine surgical margins, destroy residual disease, and to detect metastatic lymph nodes have been revisited with imaging, rapid PCR testing, intraoperative photodynamic therapy, and sentinel lymph node mapping. Immunotherapeutic approaches to alter perioperative immunosuppression to limit recurrences may have important prophylactic effects. ${ }^{10}$ Finally, postoperative therapies need to be explored and revisited with the medical and radiation oncology community. This provides ample evidence, and more importantly an opportunity, for surgeons to dedicate efforts in ongoing investigations in preventing postoperative recurrences. Advancements aimed at recurrence elimination would have a tremendous impact and improve the lives of more than 100,000 individuals each year in the United States alone.

ACKNOWLEDGMENT L.A. was supported from the Lavin Family Supporting Foundation. J.P. was supported by a Doris Duke Charitable Foundation Research Grant. S.S. was supported by the National Institutes of Health (K12CA076931) and American Cancer Society (\#IRG-78-002-30).

\section{REFERENCES}

1. Sugimura H, Nichols FC, Yang P, et al. Survival after recurrent non-small-cell lung cancer after complete pulmonary resection. Ann Thorac Surg. 2007;83(2):409-17; discussion 417-8.

2. Kelsey CR, Marks LB, Hollis D, et al. Local recurrence after surgery for early stage lung cancer: an 11-year experience with 975 patients. Cancer. 2009;115(22):5218-27.

3. Tsikitis VL, Malireddy K, Green EA, et al. Postoperative surveillance recommendations for early stage colon cancer based on results from the clinical outcomes of surgical therapy trial. J Clin Oncol. 2009;27(22):3671-6.

4. Guyot F, Faivre J, Manfredi S, et al. Time trends in the treatment and survival of recurrences from colorectal cancer. Ann Oncol. 2005;16(5):756-61.

5. Voduc KD, Cheang MC, Tyldesley S, et al. Breast cancer subtypes and the risk of local and regional relapse. J Clin Oncol. 2010;28(10):1684-91.

6. Giordano SH, Buzdar AU, Smith TL, et al. Is breast cancer survival improving? Cancer. 2004;100(1):44-52.

7. Imkampe A, Bendall S, Bates T. The significance of the site of recurrence to subsequent breast cancer survival. Eur J Surg Oncol. 2007;33(4):420-3.

8. Agarwal PK, Sadetsky N, Konety BR, et al. Treatment failure after primary and salvage therapy for prostate cancer: likelihood, patterns of care, and outcomes. Cancer. 2008;112(2):307-14.

9. Freedland SJ, Humphreys EB, Mangold LA, et al. Risk of prostate cancer-specific mortality following biochemical recurrence after radical prostatectomy. JAMA. 2005;294(4):433-9.

10. Shakhar G, Ben-Eliyahu S. Potential prophylactic measures against postoperative immunosuppression: could they reduce recurrence rates in oncological patients? Ann Surg Oncol. 2003;10(8):972-92. 